\title{
FORMACIÓN PROFESIONAL EN ENFERMERÍA BASADA EN EL CUIDADO HUMANO
}

\author{
NURSING EDUCATION BASED ON HUMAN CARE \\ DORIS GONZÁLES CARHUAJULCA \\ Universidad Científica del Sur
}

FLOR LUNA VICTORIA MORI

Universidad Nacional de Trujillo

\section{RESUMEN}

La presente investigación tuvo como objetivo evaluar la integración de la Teoría del Cuidado Humano en la formación profesional universitaria en los estudios de Enfermería. Para ello se consideraron las teorías sobre cuidado humano de Milton Mayeroff y Jean Watson, así como las experiencias de formación profesional en una universidad privada de la ciudad de Chiclayo (Perú) durante el primer semestre de 2018. Se realizó un estudio descriptivo con enfoque mixto secuencial. Se aplicaron entrevistas semiestructuradas a seis docentes de especialidad y encuestas a noventa y tres estudiantes de los dos últimos ciclos de estudio. Las entrevistas fueron procesadas a través del programa Atlas Ti, y las encuestas, a través de estadística simple con el programa excel. Se concluyó que los principios de la teoría del cuidado humano en el proceso de formación profesional universitaria en los estudios de Enfermería no habían sido integrados.

Palabras clave: formación universitaria, cuidado humano, enfermería, métodos mixtos.

\section{ABSTRACT}

The objective of this research was to evaluate the inclusion of the Theory of Human Caring in the professional development of Nursing students. In order to do so, we considered Milton Mayeroff and Jean Watson's theories about human care and experiences about professional education of a private university in Chiclayo (Peru) during the first semester in 2018.

Correspondencia: dgonzalesc@científica.edu.pe 
We conducted a descriptive study with a sequential mixed approach. We used semistructured interviews with six nursing professors and applied surveys to 93 students enrolled in their last two academic terms: the interviews were processed through Atlas Ti software and the surveys, through simple statistics with Excel.

It was concluded that the principles of the theory of human caring had not been integrated in the university education process.

Key words: university education, Human Care, Nursing, mixed methods.

\section{INTRODUCCIÓN}

El Sistema Nacional de Evaluación, Acreditación y Certificación de la Calidad Educativa (SINEACE) informó en 2018 que hasta entonces 13 escuelas y facultades de Enfermería lograron el proceso de acreditación. De ellas, solo dos hacen referencia al cuidado centrado en el ser humano: la ex Escuela de Enfermería Padre Luis Teza, de la Universidad Ricardo Palma (Lima), y la Escuela de la Universidad Católica Santo Toribio de Mogrovejo (Chiclayo), ambas con la particularidad religiosa como paradigma central institucional.

En cuanto a las cuestiones curriculares y las prácticas pedagógicas o de enseñanza-aprendizaje en las ciencias de enfermería, Waldow (2009) identificó dos grandes características: entorno y profesorado-alumnado. Sobre el entorno, dijo que el profesor es responsable de proveer una atmósfera amigable y segura para el aprendizaje, y que esta puede extenderse a todo el medio académico para sensibilizar a otros docentes. Sobre el profesorado-alumnado, se subraya la necesidad de enseñar y aprender juntos, cobrando especial interés tanto el rol docente como las posibilidades de desempeño de dicho rol. En una enseñanza centrada en el cuidado, tanto la clase, el laboratorio, como el campo clínico tornan lugares de encuentro donde los alumnos son copartícipes en las actividades de aprendizaje; sus opiniones y sugerencias son bienvenidas, así como las del equipo de enfermería del campo clínico.

Respecto a la formación profesional universitaria, Cuéllar (2007) advierte que el término formación ofrece infinidad de posibilidades conceptuales asociadas a la acción educativa. Se vincula al concepto de dar forma, moldear, transformar, con-formar y de-formar, está directamente relacionado con un actuar que se ejerce sobre determinado objeto y que le da una nueva forma a una misma esencia; asimismo, manifiesta unas características diferentes a las originales.

Horruitiner (2006) agrega al concepto de formación el término preparación integral. Refiere que en la educación superior, la formación se caracteriza por ser un proceso sustantivo desarrollado en las universidades con el objetivo de preparar integralmente al estudiante en una determinada carrera universitaria, y comprende tanto los estudios de pregrado o de grado, como también los de posgrado. Freire (2011) habla de una pedagogía de la libertad; señala que la formación es un modo de educar, una verdadera reflexión y acción del hombre sobre el mundo para transformarlo. 
Por otra parte, Horruitiner (2006) sostiene que las tres dimensiones consideradas para el proceso de formación profesional son la instructiva, la educativa y la desarrolladora. La dimensión instructiva es la que dota al estudiante de conocimientos y habilidades esenciales de su profesión, y lo preparan para hacer uso de ellas al momento de desenvolverse como profesional en un puesto de trabajo. Esta dimensión está compuesta por dos indicadores: demuestra conocimientos referentes a su profesión y demuestra habilidades.

La dimensión desarrolladora está fundamentada en el vínculo que debe existir entre el estudio y el trabajo. Para que un estudiante pueda desempeñarse en el mundo laboral no bastará con tener conocimientos y habilidades, es también indispensable la actualización de dicha instrucción en relación con el estado actual del desarrollo de la profesión, y esto se logrará al establecer contacto con el objeto de su profesión desde los primeros años de su carrera.

En cuanto a la base conceptual del cuidado humano, Mayeroff (1990) afirma que cuidar no es una relación momentánea, sino un proceso de ayudar a otro a crecer y actualizarse a través de la confianza mutua y mediante la profundización y la transformación cualitativa de la relación. Además, indica que la esencia del cuidado se basa en el profundo respeto por la otra persona, que constituye un componente crucial y vital en la enfermería. En tal sentido, propone ocho pasos que deben considerarse para dar un cuidado humanizado: obtener conocimientos, alternar ritmos, paciencia, honestidad, confianza, humildad, esperanza y coraje.

En la misma línea, Watson (2011) sostiene que el cuidar es parte fundamental del ser y es el acto más primitivo que un ser humano realiza para, efectivamente, llegar a ser; y este ser es precisamente un ser en relación a otro que lo invoca. Este llamado desde el otro es la base fundadora del cuidado en general y, a la vez, del cuidado profesional propio de la enfermería. Este cuidado profesional es estructurado, formalizado y destinado a satisfacer las necesidades del ser humano con el propósito de promover, mantener o recuperar la salud.

Watson basa su teoría en diez factores de cuidados o factores caritativos de cuidados: formación humanista-altruista en un sistema de valores; ser auténticamente presente, y permitir y mantener el sistema de creencias profundas y subjetivas del individuo, compatible con su libertad; el cultivo libre de las propias prácticas espirituales y transpersonales, que vaya más allá del ego y la apertura a los demás con sensibilidad y compasión; desarrollo y mantenimiento de una auténtica relación de cuidado, a través de una relación de confianza; permitir la expresión de sentimientos positivos y negativos: escuchar verdaderamente la historia de la otra persona; la utilización sistemática del método científico de resolución de problemas durante el proceso de toma de decisiones; la promoción de una enseñanza-aprendizaje interpersonal; la creación de un medio ambiente mental, físico, sociocultural y espiritual de sostén, de protección y de corrección; la asistencia en la satisfacción de las necesidades humanas; y el tomar en consideración los factores existenciales-fenomenológicos.

Una de las manifestaciones de haber recibido una atención de calidad cuando se atiende en algún servicio de salud es el buen cuidado profesional recibido; intentar medir esta percepción no es fácil por el 
grado de subjetividad que invadiría cualquier investigación.

Orozco y Vija (2018) sostienen que los pacientes valoran el afecto y el buen trato, como factores de atención de calidad. Consideran que ello no se logra principalmente por la sobrecarga laboral del personal de enfermería, entre actividades propias de su profesión y otras administrativas, que las instituciones de salud les exigen. Sin embargo, Monchón y Montoya (2014) afirman que el 64,18\% de los usuarios en el Hospital Almanzor Aguinaga Asenjo de Chiclayo (Perú) indican que el nivel de calidad del servicio de enfermería es bajo, especialmente en referencia a la pérdida de la intimidad del paciente y la incapacidad para entablar una relación de confianza ni con el paciente ni con el familiar. En la misma línea, Martínez (2018) indica que la percepción de los adultos mayores respecto al trato humanizado del personal de enfermería varía según el tipo de centro de salud y el tiempo de hospitalización; los pacientes hospitalizados en un centro de salud público durante más tiempo tienen una percepción desfavorable.

La presente investigación consideró una sola variable: formación profesional universitaria de Enfermería basada en el cuidado humano, entendiéndola como el conjunto de acciones sistematizadas que se dirigen al estudiante en la totalidad de su ser y vinculado a su entorno físico, social y cultural, con el propósito de transformar su esencia mediante sólidos conocimientos de las ciencias, con un compromiso con la sociedad y el medio ambiente. Así mismo, se considera que el proceso de formación debe estar ligado a una formación en valores y aptitudes que promuevan la defensa de la vida y el respeto a la dignidad de cada ser humano, familia y comunidad que se atienda.

Ante esta problemática se planteó la siguiente pregunta de investigación: ¿Cómo se ha integrado el cuidado humano en la formación universitaria en los estudios de Enfermería en Chiclayo (Perú)?, con la hipótesis de que existen deficiencias en la integración de la Teoría del Cuidado Humano en la formación profesional de Enfermería. En consecuencia, el objetivo general de este estudio fue evaluar el proceso de integración del cuidado humano en la formación universitaria en los estudios de Enfermería en una universidad de Chiclayo (Perú).

\section{MATERIAL Y MÉTODOS}

Estudio observacional, descriptivo en el que se utilizó métodos mixtos. Se consideró el diseño mixto de Triangulación Concurrente (DITRIAC) porque permite comparar y contrastar los datos originados por estas distintas metodologías, según Hernández (2014).

Los datos se investigaron desde una perspectiva de tipo descriptiva no experimental y transversal. El fenómeno observado fue la formación profesional universitaria en Enfermería. Para ello se aplicó un cuestionario a estudiantes del último año de Enfermería durante el primer semestre del 2018, cuando iniciaron el curso de internado; asimismo, se utilizó una entrevista semiestructurada aplicada a las enfermeras docentes de las asignaturas de especialidad que participaron en la formación de los estudios de Enfermería en el primer semestre de 2018. Se aplicó el cuestionario a 93 estudiantes del último año: 37 de noveno ciclo y 56 de décimo ciclo (en el Perú, la 
licenciatura en Enfermería exige el estudio de 10 ciclos); la entrevista se aplicó a 6 docentes coordinadores de los cursos de especialidad que trabajaron en ese semestre.

El cuestionario se construyó en base a tres dimensiones: dimensión instructiva con los indicadores "demuestra habilidades" y "demuestra conocimiento"; dimensión desarrolladora, con los indicadores "capacidades instructivas" y "responsabilidad social"; y dimensión educativa, con un único indicador "cualidades personales". Posterior a la identificación de las dimensiones se establecieron los criterios de medición, a partir de la integración de los conceptos de educación por competencias, según Horruitiner, y con los 10 procesos caritas del cuidado humano de Watson. Los instrumentos utilizados en la presente investigación fueron validados por dos métodos: el juicio de expertos y el análisis estadístico del Alfa de crombach, posterior a la aplicación de la prueba piloto para el cuestionario. Para la entrevista también se realizó una prueba piloto y las respuestas transcritas fueron sometidas a un juicio de expertos.

El cuestionario aplicado consta de introducción, instrucciones y 30 preguntas cerradas sobre aspectos de la formación profesional universitaria en Enfermería basada en el Cuidado Humano. Las puntuaciones de las respuestas son como sigue:

\begin{tabular}{ll}
\hline Excelente & 4 \\
Bueno & 3 \\
Regular & 2 \\
Necesito mejorar & 1 \\
\hline
\end{tabular}

La recolección de datos cuantitativos se realizó después de la clase teórica de la asignatura de Seminario de Tesis. Se les ex- plicó a todos los alumnos los objetivos de la investigación, el desarrollo del contenido y se repartió el consentimiento informado para confirmar su aceptación. Este procedicimiento fue para ambos ciclos, noveno y décimo, y se realizó con el permiso de la dirección de la Escuela de Enfermería. Para la recolección de datos cualitativos se eligió a las docentes coordinadoras que dictaban la teoría de los cursos de especialidad, pues ellas habían participado del proceso de formación de las estudiantes antes encuestadas. El tiempo de recolección de ambos datos se realizó en aproximadamente 4 meses; la entrevista a las docentes tomó más tiempo debido a los diversos horarios de trabajo tanto en la universidad como en sus centros hospitalarios.

Para analizar los resultados del cuestionario se utilizó el programa SPSS Software de Procesamiento Estadístico versión 25 con estadística simple. Para analizar la información de las entrevistas semiestructuradas se utilizó el programa Atlas TI y con ello se realizó el análisis de contenido. De acuerdo con Polit (2000), esto permite describir y analizar la realidad para diseñar una propuesta que encamine a la transformación de la formación profesional universitaria para la carrera de Enfermería basada en el cuidado humano.

Durante la investigación se siguió los siguientes criterios de rigor científico:

Credibilidad, pues los resultados de la presente investigación partieron de la recolección de datos e información de las propias fuentes primarias, mediante la aplicación de los instrumentos de recolección de datos, rescatándose el valor de la verdad. Auditabilidad o confirmabilidad, pues en el presente trabajo se examinaron datos e informaciones anteriores considera- 
das dentro de los antecedentes, las cuales nos ayudaron a orientar la investigación. Transferibilidad o aplicabilidad, pues los resultados de la presente investigación proporcionaron información importante para ser tomada como punto referencia en investigaciones posteriores con una temática relacionada.

En cuanto a los principios bioéticos, se tuvo en cuenta el principio de autonomía, el cual les dio la posibilidad a los participantes de decidir aceptar o abstenerse de participar en el presente estudio, decidir el mejor momento para la aplicación de los intrumentos y hacer pausas o detener las entrevistas en el momento que lo solicitaran; el principio de beneficencia permitió tener en cuenta los sentimientos, percepciones y silencios durante las entrevistas; además, se respetó la participación durante ella, evitando hacer comentarios que pudieran dañar o interferir en los discursos dados; y el principio de justicia, pues se les dio el mismo trato a todos los participantes.

\section{RESULTADOS}

Posterior al procesamiento de los datos de las encuestas, se representaron gráficamente los resultados de la medición de los indicadores, integrando los criterios por indicador de cada una de las 3 dimensiones de la formación profesional universitaria de Enfermería basada en el cuidado humano.

En la Figura 1 se detallan los criterios del indicador que mide las habilidades para la profesión, correspondiente a la dimensión instructiva de la formación profesional de Enfermería basada en el cuidado humano. Los resultados muestran un nivel bueno de las estudiantes en la aplicación del método de enfermería (3.15/4.00) y en la administración de fármacos (3.45/4.00); sin embargo, sus niveles de uso de las TIC, capacidad de búsqueda de información y argumentación, así como sus habilidades para la aplicación de técnicas terapéuticas, solo son regulares (menores e iguales a 3.00 de un máximo de 4.00).

FIGURA 1

Dimensión instructiva: demuestra habilidades para la profesión

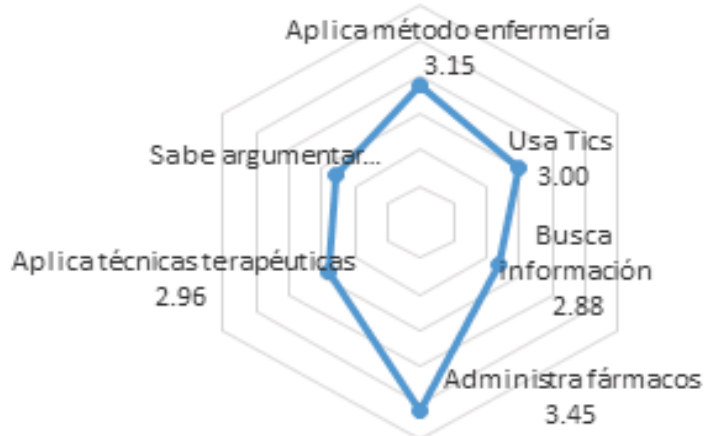

Encuesta realizada a estudiantes del último ańo de la carrera de Enfermería de una universidad privada de Chiclayo 2018. 
En la Figura 2 se observan los criterios del indicador que mide los conocimientos sobre la profesión, respecto a la dimensión instructiva de la formación profesional de En- fermería basada en el Cuidado Humano. Los resultados muestran en promedio un nivel bueno; sin embargo, el indicador de uso de metodología científica $(2.86 / 4.00)$ es regular.

\section{FIGURA 2}

Dimensión instructiva: demuestra conocimientos de la profesión

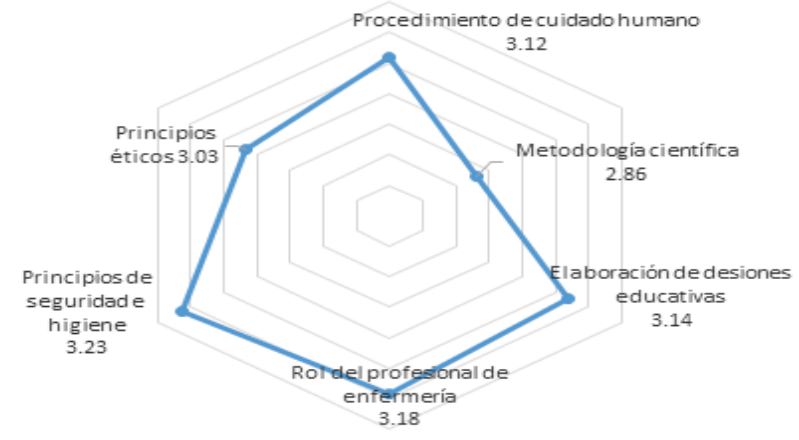

En la Figura 3 se observan los criterios correspondientes al indicador que mide la capacidad instructiva en contexto de la profesión, correspondiente a la dimensión desarrolladora de la formación profesional de Enfermería basada en el cuidado hu- mano. El valor más elevado se encuentra en el indicador referido a la comunicación de información de salud (3.18/4.00); sin embargo, poseer una cultura emprendedora $(2.83 / 4.00)$ y el uso de la investigación (2.91/4.00) presentan un nivel regular.

FIGURA 3

Dimensión desarrolladora: capacidad instructiva en contexto de la profesión

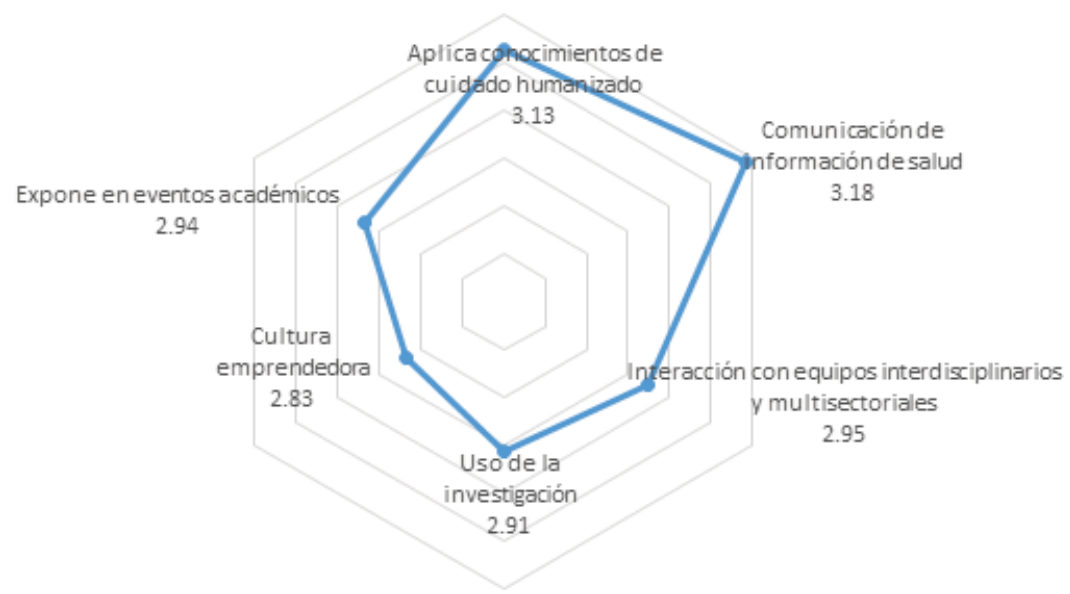


En la Figura 4 se detallan los criterios del indicador que mide la responsabilidad social para la profesión, correspondiente a la dimensión desarrolladora de la formación profesional de Enfermería basada en el Cuidado Humano. Se observa como promedio un nivel bueno; el respeto a la diversidad cultural es el más elevado (3.18/4.00).

\section{FIGURA 4}

\section{Dimensión desarrolladora: demuestra responsabilidad social}

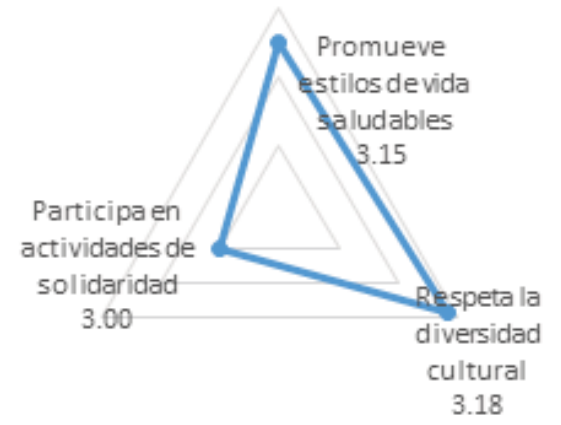

En la Figura 5 se detallan los criterios inherentes al indicador que mide las cualidades personales, correspondiente a la dimensión educativa de la formación profesional de Enfermería basada en el cuidado humano. Los resultados muestran en promedio un nivel bueno; los más altos son el respeto a las necesidades espirituales del paciente $(3.33 / 4.00)$ y el respeto el derecho a la vida (3.32/4.00). Los factores con menos valores son el respeto a códigos éticos y legales (3.20/4.00) y el compromiso con el paciente y sus familiares en situaciones críticas (3.22/4.00).

\section{FIGURA 5}

\section{Dimensión educativa: cualidades personales}

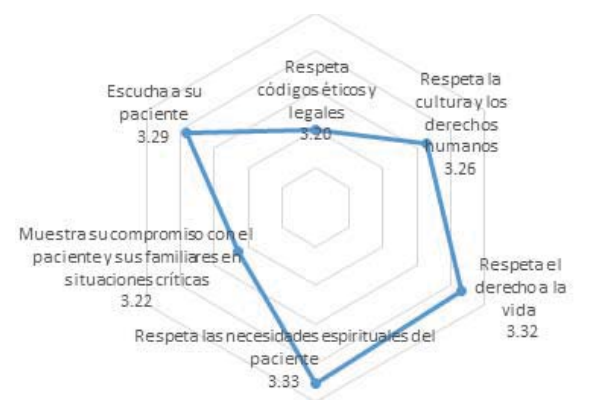


En relación al análisis de los contenidos de las entrevistas, se definieron las siguientes categorías por dimensión:

Dimensión Instructiva: transversalidad curricular, integración silábica, evaluación integral e idoneidad docente. En relación a la transversalidad curricular, las docentes manifiestan que las teorías del cuidado humano solo se integran en algunos cursos de especialidad; no es secuencial a lo largo del plan de estudios. En relación a la integración silábica manifiestan: «Fácilmente los docentes podemos consignarlo dentro de los sílabos, sin embargo, considero que se nos debe capacitar para hacer efectiva la metodología». Respecto a la evaluación integral, manifiestan: "Durante la evaluación de las asignaturas los instrumentos aplicados no incorporaron dichos elementos. En algunas asignaturas centrales solo es un tema». Respecto a la idoneidad docente manifiestan que existe incoherencia entre lo que algunas profesoras expresan verbalmente y lo que hacen en la práctica, no inspiran confianza.

Dimensión Desarrolladora: visión antropológica, coherencia pedagógica y evaluación, atención clínica y comunitaria. En relación a la visión antropológica manifiestan: "Como docentes nos enfocamos, en muchas ocasiones, en que el estudiante aprenda todos los procedimientos, normas técnicas, términos científicos y prácticos y dejamos de lado la inclusión del cuidado humano, que en mi opinión debe ser la clave principal para brindar un cuidado integral". Respecto a la coherencia pedagógica manifiestan: «Cabe resaltar que las docentes de práctica laboran más de 20 años como profesionales de enfermería y ello convierte el cuidado en una rutina práctica, mis- ma que es observada por los estudiantes y contradice la teoría, por ello escucho tantos comentarios que manifiestan los estudiantes como "nos hablan del cuidado humano, pero el trato de la docente es déspota", "en el hospital no existe el cuidado humano por parte los profesionales de la salud", "la teoría no se vincula con la realidad". Respecto a la evaluación, opinan que se deben establecer los instrumentos de evaluación para el proceso de enseñanza-aprendizaje y para la atención clínica y comunitaria.

Dimensión Educativa: valores, cualidades y virtudes. En relación a esta dimensión, las docentes consideran que se integra el cuidado humano aplicando competencias asociadas a valores profesionales y sentimientos como de amor, amistad y empatía, además de brindar el conocimiento científico teórico en combinación con el conocimiento práctico.

\section{DISCUSIÓN}

En relación con la dimensión instructiva, que está referida a los conocimientos y habilidades del estudiante en relación a su profesión, la presente investigación demuestra que los estudiantes tienen un nivel bueno de conocimientos, pero el desarrollo de sus habilidades solo es regular, sobre todo en actividades no cotidianas, como la investigación y las técnicas terapéuticas, en las cuales se requiere una acción más autónoma y crítica del estudiante. Ello advierte que los estudiantes no han sido preparados, como señala Waldow (2009), para ser copartícipes en las actividades de aprendizaje ni a problematizar, de forma crítica, las cuestiones de salud.

Hay gran posibilidad de que no tengan la predisposición, como señalan Muñoz et 
al. (2009), de generar una empatía con los pacientes y familiares, y brindarle un ambiente de confianza en donde el paciente pueda expresar sus sentimientos y sentir calidez del servicio del personal de enfermería.

Según las entrevistas realizadas a docentes, manifiestan que no hay una integración efectiva del cuidado humano ni en las sesiones de aprendizaje ni en los instrumentos de evaluación, considerando que debe ser un tema transversal en el currículo. Sostienen que no existe una metodología de integración del cuidado humano en la formación profesional; sin embargo, reconocen la importancia de ello. El diseño metodológico de las sesiones de aprendizaje se enfoca más en lo cognitivo y procedimental, dejando de lado lo humanístico. Coinciden en que el personal docente debe tener vocación humana; en algunos no se percibe el cuidado humano en sus actos, tanto en las clases teóricas como en las prácticas en los centros de salud.

En relación a la dimensión desarrolladora, que está fundamentada en el vínculo que debe existir entre el estudio y el trabajo, la investigación demuestra que los estudiantes son solidarios, participan en actividades de responsabilidad social, pero no tienen iniciativas autónomas como la investigación y el emprendimiento; presentan carencias para exponer en público e interactuar con otros actores del medio. Ello les limita para generar ideas, crear espacios y estrategias para integrar a los pacientes y sus familiares, y hacerles sentir que le ofrecen un trato personalizado. Se coincide con León (2013) y Acevedo y Alvarado (2015) en que los estudiantes sí perciben la bondad e importancia del cuidado humano involucrado en su proceso de aprendizaje, participando de ello en forma activa, siempre que no sean quienes lideren el proceso; pero no son capaces de tener la iniciativa de hacerlo y en la medida que en los últimos ciclos los estudiante tienen menor acompañamiento de los docentes y son involucrados en responsabilidades fuera de la universidad y sin la compañía de ellos, prefieren cumplir sus actividades cotidianas y no verse involucrados más de lo necesario con el paciente. Al respecto, los docentes sostienen que existe incoherencia entre lo aprendido en clase y lo observado en las prácticas; el desempeño de los profesionales de enfermería está influenciado por la rutina y se carece de instrumentos de evaluación de la atención clínica y comunitaria que permitan detectar esas irregularidades y mejorar. La aplicación de cuidado humano establece concebir el enfoque que va a proyectar el docente durante la práctica, es decir, va a usar los conocimientos en fisiología, fármacos, entre otros, que son ciencias universales del cuidado, e incorporar la visión antropológica de la persona.

En relación a la dimensión educativa, constituida por las cualidades y valores en la personalidad del estudiante, los resultaron demuestran que los estudiantes sí tienen la voluntad de ayudar a los pacientes. En ello hay plena coincidencia con Waldow (2009), Muñoz et al. (2009), León (2013) y Acevedo y Alvarado (2015). En relación a ello, los docentes coinciden en que los estudiantes evidencian solidaridad y opinan que se deben generar valores, cualidades y virtudes desde las sesiones de aprendizaje en las relaciones docente-estudiante y estudiante-estudiante. Valores como respeto, responsabilidad, honestidad y compromiso; cualidades como facilidad de comunicación, capacidad para relacionarse con los demás, capacidad de análisis, liderazgo, trabajo en equipo y creatividad; virtudes como tolerancia, empatía, confianza, prudencia y justicia. 
El estudio presenta una serie de limitaciones que deben considerarse. Se realizó solo en un centro de educación superior, una universidad privada, y por tanto solo contempla una realidad. La muestra fue intencionada por la falta de disponibilidad de algunas enfermeras docentes y el acceso limitado para encuestar a los estudiantes.

\section{CONCLUSIONES}

Los estudiantes observados en esta investigación tienen una formación humana coincidente con lo expresado por Mayeroff y Watson; no obstante, falta una mayor interrelación entre la teoría del cuidado humano, consignados por los dos autores y los diversos elementos del proceso de aprendizaje: competencia docente en cuidado humano, diseño de sesiones de aprendizaje con énfasis en cuidado humano, desarrollo de habilidades relacionadas con el cuidado humano en actividades en centros de salud con pacientes, instrumentos de evaluación que sean pertinentes con la competencia basada en el cuidado humano y puedan evaluar integralmente al estudiante para lograr un buen profesional y una buena persona a la vez, con sentido crítico, emprendedor y con habilidades investigativas desarrolladas.

Por tanto, se concluyó que los principios de la teoría del cuidado humano en el proceso de formación profesional universitaria en los estudios de Enfermería no habían sido integrados.

Se recomienda plantear un nuevo modelo de formación profesional universitaria para los estudios de Enfermería basado en el cuidado humano. Asimismo, se hace necesario diseñar una estrategia para implementar el nuevo modelo y sus instrumen- tos de mejora continua. Por último, sería conveniente realizar un estudio similar en estudiantes de Enfermería de una universidad pública, para establecer diferencias con las universidades privadas, o incluso establecer una red de docentes investigadores con el objetivo de evaluar la implementación de este proceso a nivel nacional.

\section{REFERENCIAS BIBLIOGRÁFICAS}

Ábela J (2003). Las Técnicas de Análisis de Contenido: Una revisión actualizada. Espańa. Centro Estudios Andaluces de la Universidad de Granada. v.10, n. 2, p. 1-34. Consulta: 29 de junio de 2017. Disponible en: http://public.centrodeestudiosandaluces.es/ pdfs/S200103.pdf.

Acevedo JC, Alvarado LC (2015). Percepción de los estudiantes de la Escuela de Enfermería Padre Luis Tezza acerca del rol docente en la formación del cuidado humanizado (Tesis licenciatura). Escuela de Enfermería Padre Luis Tezza. Lima, Perú.

Castillo E (2003). El rigor metodológico en la Investigación Cualitativa. Colombia. Corporación Colombia Médica del Valle. (34), $164-167$.

Cuéllar Z (2007). Hacia un currículo vivo: la evaluación en educación médica. Educación y Educadores. 10(1), 89-103.

Escuela de Enfermería Padre Luis Tezza de la Universidad Ricardo Palma (2018). [Internet]. Consulta: 3 de diciembre de 2018. Disponible en: http://www. eeplt.edu.pe/mision-vision-valores/.

Escuela de Enfermería de la Universidad Privada Antenor Orrego (2018). [Internet]. Consulta: 3 de diciembre de 2018, de: http://www.upao.edu.pe/ facultades/index.aspx?mod=mod_esc\&e=ENFE. 
Freire P (2011). La educación como práctica de la libertad. México. Siglo Veintiuno Editores.

Hernández R, Fernández C, Baptista M (2014). Metodología de la investigación. Ciudad de México, México. Mc Graw Hill. 5º edición.

Horruitiner P (2006). La Universidad Cubana: el modelo de formación. La Habana, Cuba. Editorial Félix Varela.

León V (2013). Percepción de los estudiantes de enfermería, sobre el cuidado humanizado de la enfermera al paciente hospitalizado (Tesis licenciatura). Facultad de Medicina, Universidad Nacional Mayor de San Marcos. Lima, Perú.

Martínez N (2018). Tipo de hospital y percepción del adulto mayor acerca del cuidado humanizado del enfermero. CASUS, 3(2), 64-71.

Mayeroff M (1990). On Caring. New York, Estados Unidos. Harper Perennial/ Harper Collins Publishers. $7^{\circ}$ edición.

Monchón P, Montoya Y (2014). Nivel de calidad del cuidado enfermero desde la percepción del usuario, servicio de emergencia, Hospital III-Essalud, Chiclayo 2013 [Tesis licenciatura]. Universidad Católica Santo Toribio de Mogrovejo. Chiclayo, Perú.

Muñoz Y, Coral R, Moreno D et al. (2009). El significado del cuidado humanizado en egresadas de la Facultad de Enfermería. Rev. Repertorio de Medicina y Cirugía. Colombia 2009; 18
(4): 246-250. Consulta: 3 de diciembre de 2018. Disponible en: http:// www.fucsalud.edu.co/sites/default/ files/2017-01/12_2.pdf.

Orozco S \& Vija M (2018). Deshumanización en la atención de enfermería hacia los pacientes en los servicios hospitalarios (Tesis licenciatura). Universidad Santo Tomás Especialización en Auditoria de Salud. Bogotá, Colombia.

Polit D, Hungler B, Palacios R, Féher G (2000). Investigación científica en ciencias de la salud: principios y métodos. México. McGraw-Hill Interamericana.

Quintero B (2001). Ética del Cuidado Humano bajo los enfoques de Milton Mayeroff y Jean Watson. Ciencia y Sociedad; 16(1):16-22. Consulta: 11 de setiembre de 2019. Disponible en: http://www.redalyc.org/ pdf/870/87011272002.pdf.

Sistema Nacional de Evaluación, Acreditación y Certificación de la Calidad Educativa. (2018) Buscador de Instituciones y carreras acreditadas [Internet]. Lima. Consulta: 2 de diciembre de 2018, Disponible en: https://app.sineace. gob.pe/buscador/acreditacion.aspx.

Waldow R (2009). Enseñanza de Enfermería centrada en el Cuidado. Bogotá, Colombia. Aquichan, 9 (9), 246-256.

Watson J (2012). Human caring Science. A Theory of Nursing. Colorado, Estados Unidos. Jones \& Bartlett Learning. 